\title{
Soldadura TIG de los aceros inoxidables dúplex del tipo 22-05 (Uranus 45N y Avesta). Estudio de la microestructura y de las propiedades mecánicas
}

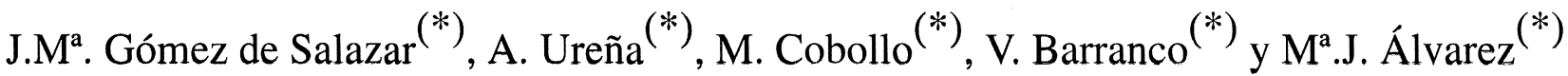

Resumen Se estudia la soldadura TIG de dos aceros inoxidables dúplex. Para ello, se ha descargado un arco sobre las chapas de material base mediante la técnica TIG, sin aportación de material y variando las E.N.A. Se realiza un estudio comparativo de la evolución microestructural, así como de las propiedades mecánicas. Se establece la relación entre los perfiles de dureza obtenidos y la variación microestructural y de la concentración de ferrita $\delta$, así comọ estas propiedades con el E.N.A. de cada cordón.

Palabras clave: Acero inoxidable dúplex. Soldadura. Microestructura. Propiedades.

\section{TIG welding of 22-05 duplex stainless steels (Uranus $45 \mathrm{~N}$ and Avesta). Microstructural studies and mechanical properties}

\begin{abstract}
TIG welding of two different duplex stainless steels is carried out. Arc-discharge on base-material plates by means of the TIG technique without filler metal and varying the energetic conditions (E.N.A.) has been performed. A comparative study concerning the microstructural evolution as well as mechanical properties is carried out. The relation between hardness profiles, the microstructural variations and the ferrita $\delta$ concentration is established. Further, the above mentioned properties are related to the E.N.A. for each welded joint.
\end{abstract}

Keywords: Duplex stainless steel. Welding. Microstructure. Mechanical properties.

\section{INTRODUCCIÓN}

Los aceros inoxidables dúplex y superdúplex comenzaron a fabricarse debido a las atractivas características que ofrecían, tanto en su comportamiento mecánico como ante la corrosión. Sin embargo, la experiencia demostró que algunas de estas aleaciones presentaban fallos estructurales al cabo de un tiempo en servicio (1). Estudios sobre el tema llevaron a la conclusión de que estos fallos se debían a serios deterioros en la zona afectada por el calor durante las operaciones de soldeo (ZAC), a causa, principalmente, de un crecimiento excesivo del grano ferrítico y de transformaciones en estado

(*) Dpto. de Ciencia de los Materiales e Ingeniería Metalúrgica. Fac. de Ciencias Químicas. Univ. Complutense de Madrid. 28040 Madrid (España). Email: gsalazar@ eucmax.sim.ucm.es sólido, tras las cuales dejaba de ser óptima la resistencia a la corrosión (2-6).

Diversos autores señalan que las técnicas de soldadura más adecuadas para este tipo de aceros son: TIG, MIG, MIG con electrodo hueco (FCAW), soldadura de arco sumergido y soldadura de arco de plasma (6 y 7).

El metal de aportación para la soldadura de aceros inoxidables dúplex muestra una matriz básicamente ferrítica con una cantidad limitada de austenita dúctil. Comparado con el metal base, el de aportación estará, por tanto, sobrealeado con níquel. Sin embargo, el metal de soldadura puede ser similar al metal base si después de la soldadura se realiza un tratamiento de recocido (1).

Los gases protectores adecuados a los procesos de soldadura aplicados a aceros inoxidables dúplex y superdúplex son el argón puro y las mezclas 
Ar- $\mathrm{O}_{2}$, o Ar-He- $\mathrm{O}_{2}$ dependiendo del proceso de soldadura empleado (1).

\section{CONDICIONES EXPERIMENTALES}

\subsection{Materiales base}

Se han estudiado dos aleaciones de aceros inoxidables dúplex del tipo 22-05: Avesta (Avesta Shieffield) y Uranus $45 \mathrm{~N}$ (Creusot-Loire), suministrados en forma de chapa laminada de 5 y $3 \mathrm{~mm}$, respectivamente. La composición química de ambas se refleja en la tabla I.

\subsection{Parámetros de soldadura}

Los parámetros de soldeo TIG utilizados en la presente investigación se recogen en la tabla II:

Los estudios realizados sobre las soldaduras han sido:

- Microscopía óptica y electrónica de barrido, con el fin de estudiar la evolución microestructural. Igualmente, se han utilizado las técnicas de análisis de imagen para determinar las dimensiones del baño fundido (B.F.) y de la ZAC en cada uno de ellos (8).

- Estudio de la variación del porcentaje de ferrita mediante ferritometría (8). Se utilizó un ferritómetro Reutlinger.

- Estudio de las variaciones de dureza, desde el centro del B.F. hasta alcanzar el material base. Las medidas se realizaron bajo norma UNE 7423-84, con un durómetro Akashi AVK-AII, aplicando una carga de indentación de $50 \mathrm{kgf}$ durante $15 \mathrm{~s}$.

\section{RESULTADOS Y DISCUSIÓN}

\subsection{Análisis microestructural. Microscopía óptica y electrónica}

\subsubsection{Evolución microestructural}

Básicamente, desde el punto de vista de la evolución microestructural que sufre cada cordón desde el centro del B.F. hasta el material base, podemos señalar que se producen los mismos fenómenos. En el centro del B.F., aparecen grandes granos ferríticos con morfología columnar. La austenita secundaria nuclea en los límites de grano ferrítico, y se desarrolla hacia el interior de la ferrita con morfología Widmanstätten. Se observa gran cantidad de picaduras en el interior de la ferrita, aunque en la aleación Avesta esto se produce con menor intensidad que en el Uranus $45 \mathrm{~N}$. Esta precipitación es de nitruros de cromo y aparece principalmente de forma intragranular en la ferrita, y en la interfase ferrita/austenita secundaria. A medida que aumenta la distancia al centro del B.F., la precipitación va disminuyendo y, paralelamente, el grano ferrítico adquiere morfologías más equiaxiales. Esto se debe a que los gradientes térmicos que se alcanzan son menores en estas, encontrándose la disipación térmica más favorecida. La ZAC es muy estrecha en este tipo de aceros, y predominan los fenómenos de recristalización y crecimiento de grano ferrítico, que adquieren forma equiaxial.

\subsection{Cálculo de las dimensiones de los cordones}

La figura 1 muestra las tendencias que siguen las dimensiones (anchura y profundidad de B.F., ZAC y total) de los cordones realizados en las dos aleaciones objeto de estudio. Se observó que la extensión de las ZAC es muy pequeña, que escasamente afecta a las dimensiones globales del cordón y en nada a la tendencia que siguen las dimensiones de los cordones al variar los parámetros de soldadura. Se observa una tendencia general en el aumento de la anchura del B.F. al aumentar la energía aportada durante la operación de soldeo en ambas aleaciones. La profundidad del baño, tanto en la aleación Avesta como en la Uranus $45 \mathrm{~N}$ sufre una estabilización y posterior descenso al aumentar la E.N.A.

\subsection{Ferritometría}

Se ha medido el contenido de ferrita, desde el centro de cada cordón hasta alcanzar el material base, cada $2 \mathrm{~mm}$. Un resumen de los resultados se muestra en la figura 2, donde se expone la evolución del contenido de ferrita de los cordones 1,4 y

TABLA I.- Composición química de las aleaciones Uranus $45 \mathrm{~N}$ y Avesta, según las especificaciones de fábrica

TABLE I.- Chemical composition of Uranus $45 \mathrm{~N}$ and Avesta alloys according to the manufacturer's specifications

\begin{tabular}{|c|c|c|c|c|c|c|c|c|c|c|}
\hline & \multirow{3}{*}{ UNS S31803 } & $\mathrm{C}$ & $\mathrm{Cr}$ & $\mathrm{Ni}$ & Mo & $\mathrm{N}$ & $\mathrm{Mn}$ & $\mathrm{Si}$ & $\mathrm{S}$ & $P$ \\
\hline Uranus $45 \mathrm{~N}$ & & 0,02 & 22,0 & 5,7 & 2,8 & 0,2 & 1,7 & 0,4 & 0,003 & 0,025 \\
\hline Avesta & & 0,017 & 21,82 & 5,72 & 3,01 & 0,155 & 1,51 & 0,34 & 0,001 & 0,022 \\
\hline
\end{tabular}


TABLA II.- Parámetros de las soldaduras efectuadas sobre los materiales estudiados

TABLE II.- Welding conditions of the parent materials

\begin{tabular}{|c|c|c|c|c|c|c|c|c|c|}
\hline Aleación & $\begin{array}{l}\text { Parámetros } \\
\text { de soldadura }\end{array}$ & $\begin{array}{c}\text { Cord. } \\
1\end{array}$ & $\begin{array}{c}\text { Cord. } \\
2\end{array}$ & $\begin{array}{c}\text { Cord. } \\
3\end{array}$ & $\begin{array}{c}\text { Cord. } \\
4\end{array}$ & $\begin{array}{l}\text { Cord. } \\
5\end{array}$ & $\begin{array}{c}\text { Cord. } \\
6\end{array}$ & $\begin{array}{c}\text { Cord. } \\
7\end{array}$ & $\begin{array}{c}\text { Cord. } \\
8\end{array}$ \\
\hline & Int. (A) & 114 & 140 & 166 & 193 & 219 & - & - & - \\
\hline AVESTA & Volt. (V) & 9,9 & 10,5 & 11 & 12,3 & 12,6 & - & - & - \\
\hline & Vel. $(\mathrm{cm} / \mathrm{min})$ & 10 & 10 & 10 & 10 & 10 & - & - & - \\
\hline URANUS & Int. (A) & 71 & 114 & 114 & 71 & 71 & 114 & 141 & 141 \\
\hline $45 \mathrm{~N}$ & Volt. (V) & 8,2 & 10,3 & 10,3 & 9,4 & 8,2 & 9,1 & 10,4 & 10,4 \\
\hline & Vel. $(\mathrm{cm} / \mathrm{min})$ & 25 & 25 & 15 & 10 & 15 & 10 & 10 & 15 \\
\hline
\end{tabular}

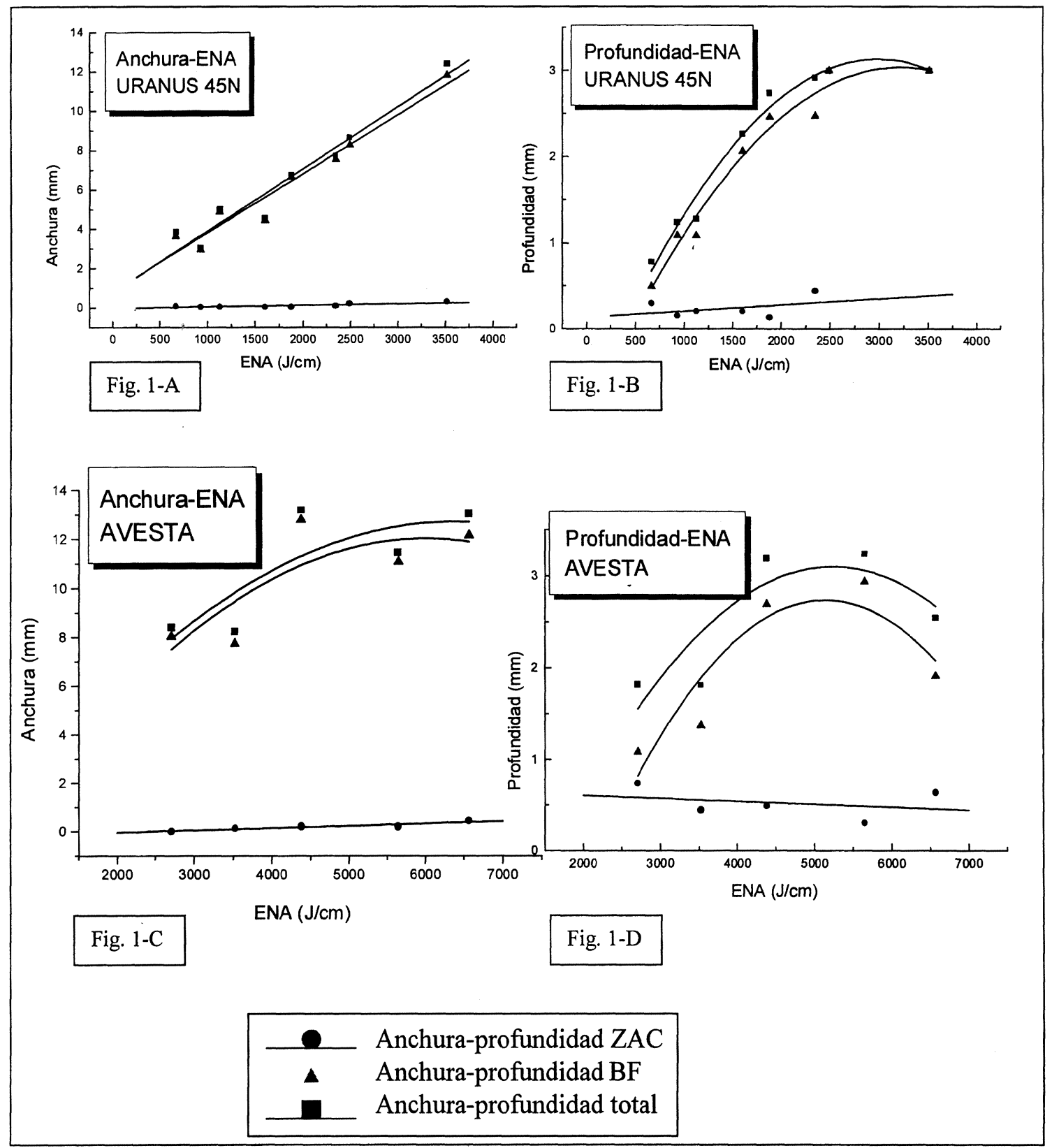

Fig. 1.- Variación de las dimensiones de los cordones en función de la energía térmica aportada (E.N.A.) en las aleaciones Avesta y Uranus 45N.

FIG. 1.- ENA vs weld dimensions of the Avesta and Uranus 45N. 


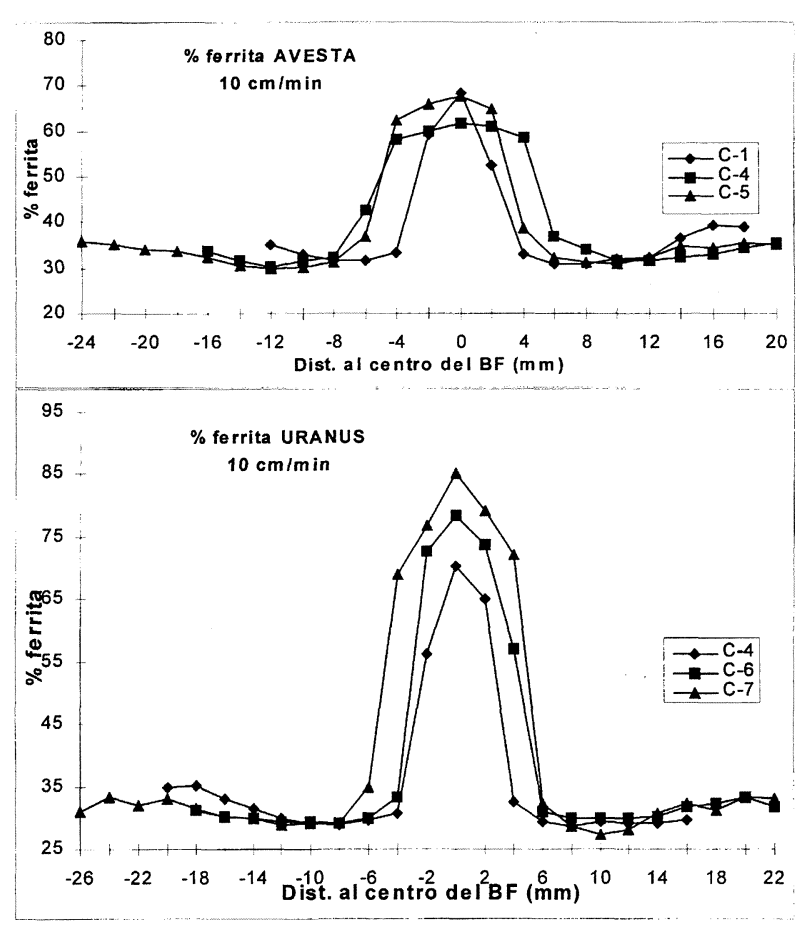

FIG. 2.- Porcentaje de ferrita en los cordones de soldadura

FIG. 2.- Ferrita content in the weld.

5 de la aleación Avesta y de los cordones 4, 6 y 7 de la aleación Uranus $45 \mathrm{~N}$. En esta figura, se observa que, para E.N.As. crecientes, la cantidad de ferrita encontrada en el centro del baño fundido en ambas aleaciones, es cada vez mayor, ya que, cuanto mayor es la temperatura alcanzada, mayor es el gradiente de temperaturas durante el enfriamiento, inhibiendo la formación de austenita.

\subsection{Ensayos de dureza}

Se llevaron a cabo dichas medidas en la cara longitudinal de los cordones, desde el centro del mismo, desplazándose a izquierda y derecha cada 2 $\mathrm{mm}$. Los resultados se exponen en la tabla III.

Como se puede deducir, en la aleación Uranus $45 \mathrm{~N}$, la dureza del material sufre una gran caída en la ZAC y B.F., recuperándose, sin embargo, hasta alcanzar valores muy superiores a los del material base en el centro del B.F. Esto indica una gran fragilización en dicha zona por la presencia de precipitados.

En el caso de la aleación Avesta, no se produce un endurecimiento tan acusado en el centro del B.F., sino que algunos cordones sufren incluso más reblandecimiento que en otras zonas. De hecho, existe una relación muy directa entre la anchura del cordón y la zona en la que se detecta el reblandecimiento del material. En cuanto a los altibajos que sufren los valores de dureza en el centro, podrían deberse, al menos en algunos cordones, al gran tamaño de grano ferrítico, que determina que las

TABLA III.- Valores de dureza Vickers encontrados a lo ancho de los cordones en las aleaciones

TABLE III.- Hardness variation across the weld

\begin{tabular}{|c|c|c|c|c|c|c|c|c|c|c|c|c|c|}
\hline & \multicolumn{8}{|c|}{ URANUS 45N } & \multicolumn{5}{|c|}{ AVESTA } \\
\hline D.a BF & $\mathrm{HV}$ & $\mathrm{HV}$ & $\mathrm{HV}$ & $\mathrm{HV}$ & $\mathrm{HV}$ & $\mathrm{HV}$ & $\mathrm{HV}$ & $\mathrm{HV}$ & $\mathrm{HV}$ & $\mathrm{HV}$ & $\mathrm{HV}$ & HV & $\mathrm{HV}$ \\
\hline$(\mathrm{mm})$ & C-1 & C-2 & C-3 & C-4 & C-5 & C-6 & C-7 & C-8 & C-1 & C-2 & C-3 & C-4 & C-5 \\
\hline-16 & & & 261 & 261 & & 273 & 263 & & & & 287 & 280 & 265 \\
\hline-14 & & 258 & 266 & 265 & 254 & 272 & 262 & 254 & & & 281 & 278 & 272 \\
\hline-12 & & 258 & 262 & 263 & 255 & 268 & 257 & 259 & 261 & 296 & 276 & 281 & 259 \\
\hline-10 & & 261 & 258 & 257 & 261 & 248 & 257 & 264 & 267 & 296 & 269 & 275 & 255 \\
\hline-8 & 253 & 266 & 254 & 253 & 262 & 264 & 256 & 259 & 265 & 290 & 262 & 271 & 255 \\
\hline-6 & 260 & 262 & 257 & 253 & 263 & 263 & 254 & 253 & 264 & 278 & 265 & 268 & 261 \\
\hline-4 & 259 & 259 & 256 & 250 & 253 & 260 & 250 & 251 & 263 & 285 & 268 & 267 & 253 \\
\hline-2 & 253 & 265 & 261 & 253 & 253 & 258 & 245 & 265 & 267 & 284 & 280 & 265 & 255 \\
\hline 0 & 269 & 271 & 258 & 257 & 270 & 258 & 270 & 250 & 247 & 257 & 256 & 275 & 279 \\
\hline 2 & 253 & 265 & 256 & 253 & 254 & 262 & 256 & 267 & 272 & 278 & 263 & 269 & 264 \\
\hline 4 & 257 & 256 & 257 & 249 & 255 & 259 & 261 & 256 & 262 & 282 & 260 & 284 & 253 \\
\hline 6 & 264 & 268 & 260 & 254 & 259 & 262 & 256 & 254 & 271 & 280 & 269 & 273 & 266 \\
\hline 8 & 259 & 267 & 256 & 255 & 258 & 268 & 259 & 258 & 280 & 284 & 272 & 270 & 262 \\
\hline 10 & 257 & 262 & 256 & 257 & 254 & 271 & 262 & 264 & 288 & 298 & 269 & 262 & 267 \\
\hline 12 & 256 & 259 & 266 & 259 & 249 & 267 & 261 & 258 & 288 & 274 & 276 & 268 & 271 \\
\hline 14 & 256 & & 265 & 264 & & 270 & 265 & 252 & 283 & 279 & 290 & 276 & 285 \\
\hline 16 & 255 & & 266 & 262 & & 277 & 268 & & 282 & & 290 & 280 & 280 \\
\hline
\end{tabular}


medidas realizadas en el centro del B.F. puedan no ser demasiado fiables. Sin embargo, sí parece existir una tendencia al aumento de la dureza en este punto, al igual que sucede con la aleación Uranus $45 \mathrm{~N}$.

\section{CONCLUSIONES}

- En la aleación Uranus 45N, en chapa de 3 mm., se consigue penetración total con aportes energéticos superiores a $2.500 \mathrm{~J} / \mathrm{cm}$. En la aleación Avesta, con un espesor de chapa de $5 \mathrm{~mm}$, no se ha conseguido penetración total con los aportes energéticos estudiados (máximo $6.500 \mathrm{~J} / \mathrm{cm}$ ), lo que indica que es más adecuada una soldadura en dos pasadas, de cara y de raíz.

- En la aleación Avesta, se consigue la máxima penetración con un E.N.A. de $4.382 \mathrm{~J} / \mathrm{cm}(3,2$ $\mathrm{mm}$ ), pero, para mayores aportes energéticos, se produce ensanchamiento del cordón sin conseguir mayor penetración.

- El máximo contenido de ferrita que se obtiene en el centro del baño fundido es, en ambas alea- ciones, en torno al $77 \%$, máximo valor aconsejado para este tipo de aceros.

\section{REFERENCIAS}

(1) Van Nassau, L., Bekkers, K., Hilkes, J. y Meelker, H. Smitweld (Lincoln Norweld, The Netherlands), Proc. Conf. 8 as J. T. de Soldadura, Adesol (Madrid), 1990.

(2) Sinha, A.K. Ferrous Physical Metallurgy. Edic. Butterworth Stoneham (MA), 1991.

(3) Solomon, H.D. y Devine, Jr. T.M. Duplex Stainless Steels. A Tale of Two Fases, En "DSS'82". Ed. R.A. Lula, ASM. Ohio 1983: 693-737.

(4) LiLjas, M. Proc. Int. Conf. Duplex Stainless Steels' 94. Glasgow (Escocia), Nov. 1994. Trabajo KV.

(5) Charles, J. Duplex Stainless Steels. Vol. 1, Ed. Les Ulis (Francia). Les Éditions de Physique, 1991, p. 151-168.

(6) Van Nassau, L., Meelker, H. y Hilkes, J. Smitweld (Lincoln Norweld, Países Bajos), Duplex Stainless Steels. Vol. 1, Ed. Les Ulis (Francia). Les Éditions de Physique, 1991: 303-323

(7) Kotecki, D.J. y Hilkes, J.L.P. Proc. Int. Conf. Duplex Stainless Steels'94. Glasgow (Escocia). Nov. 1994. Trabajo KVI.

(8) Elmer, J.W. y Eagar, T.W. Rev. Weld. Res., Supp. Weld. J. Abr. 1990: 141-150. 